\title{
Cuadro de Mando Integral (BSC) del Servicio de Pediatría del Hospital Regional Rancagua
}

\author{
HEIDY LEIVA ${ }^{(1)}$ y MATÍAS FLAMM ${ }^{(2)}$
}

\section{RESUMEN}

El cuadro de mando integral (BSC) es un modelo de gestión estratégico-operacional que permite desarrollar, implementar y controlar la aplicación de una estrategia en una organización. Se presenta el desarrollo del modelo en el Servicio de Pediatría del Hospital Regional de Rancagua y su evaluación, luego de 4 años de aplicación plena. Es posible demostrar que esta metodología claramente recomendada en las empresas privadas es susceptible de aplicar exitosamente en un servicio clínico de un hospital público. La implementación del modelo significó que todo el equipo de salud del servicio internalizara y se comprometiera con la gestión alcanzando las metas propuestas en forma participativa en el período evaluado.

Palabras clave: gestión, sistema de salud público

\section{ABSTRACT}

BALANCED SCORECARD (BSC) AT THE DEPARTMENT OF PEDIATRICS OF THE RANCAGUA REGIONAL HOSPITAL

Balanced Scorecard is an operational-strategic management model that enables the development, implementation and control of the application of a strategy within an organization. The present work introduces the development of the model in the Department of Pediatrics of the Rancagua Regional Hospital and its assessment after 4 years of its full application. It is possible to demonstrate that such methodology, clearly recommended among private corporations, may be successfully applied in the clinical department of a public hospital. The implementation of the model meant that all the health care team in the department internalized the management and committed to it, attaining the proposed goals participatively throughout the assessment period.

Keyword: management, public health care system

\section{INTRODUCCIÓN}

En el ámbito de la gestión y planificación, el sector público del sistema de salud en general y los médicos en particular, han sido criticados, entre otras causas porque no se aprecia una actitud de evaluación y búsqueda del mejor costo-efectividad, lo que ha motivado, con frecuencia, un intento de los directivos de im- poner formas de gestionar que sólo han tensionado a las organizaciones sin que se aprecie el convencimiento de los actores de la necesidad de introducir metodología de gestión. Parece lejana al sentir del médico y del equipo de salud, la aplicación de términos como productividad o eficiencia en un arte como la Medicina en que el sujeto de trabajo tiene extrema variabilidad y los procesos, en consecuencia no son rígidos.

(1) Servicio de Pediatría. Hospital Regional Rancagua. Alameda 61, 6º Piso. Rancagua. Chile. draheidy@terra.cl

(2) Escuela de Medicina. Facultad de Ciencias de la Salud. Universidad de Santiago de Chile. Santiago. Chile. 
Por otra parte, el proceso productivo puede resultar en un resultado negativo dado la condición de mortalidad inherente al ser humano. Es decir, el negocio de la atención de salud tiene incorporado que el resultado final es inevitable en condiciones de extrema complejidad en que el paciente se puede encontrar fuera de alcance terapéutico.

Aún así, es un imperativo ético de todo el equipo de salud utilizar los recursos de la mejor forma y en nuestro servicio demostramos que este modelo es factible de utilizar en la gestión de una empresa pública, como es el Servicio de Pediatría del Hospital Regional Rancagua que debe responder a las nuevas exigencias incorporadas por la reforma de salud

El balanced scorecard (BSC) ${ }^{1}$ o cuadro de mando integral representa un modelo de gestión estratégico-operacional que permite desarrollar, comunicar e implementar una estrategia, posibilitando la obtención de resultados a corto y mediano plazo.

Este modelo utiliza elementos de otros modelos de gestión y permite dar una mirada global y nueva a la organización ${ }^{2-5}$.

El modelo considera el sistema causa efecto de la calidad total, la excelencia de los procesos de la reingeniería, el valioso e irremplazable aporte del recurso humano del concepto de empowerment, la influencia del entorno y cadena de valor de Porter y las declaraciones estratégicas de Visión, Misión y Valores.

Con las variables internalizadas, se configura el modelo que se compone de un sistema de relaciones causales entre objetivos declarados en 4 perspectivas, financiera, de clientes, de procesos y de personas, para alcanzar el norte estratégico, que es la Visión, considerando las fuerzas internas y externas que influyen en la organización. A todo lo anterior, el modelo asocia una serie de indicadores con el objetivo de monitorear la gestión y el cumplimiento de las actividades planificadas. Las iniciativas estratégicas o planes de acción contribuyen a lograr las metas declaradas, cuya selección, priorización y asignación de recursos específicos es fundamental en los mapas estratégicos. Entendiendo como meta aquel valor que se desea para un indicador en un período determinado En síntesis, el modelo está compuesto por:

- Las declaraciones estratégicas corporativas que marcan el rumbo de la organización.

- Un conjunto de objetivos estratégicos dispuestos en 4 perspectivas.

- Un conjunto de indicadores asociados a los objetivos.

- Una direccionalidad de referencia hacia donde se pretende llevar o movilizar cada indicador.

- Un conjunto de acciones a realizar para alcanzar el valor esperado de cada indicador.

Figura 1. Modelo Cuadro de Mando Integral. Las 4 perspectivas.

\section{PERSPECTIVA \\ PERSONAS}

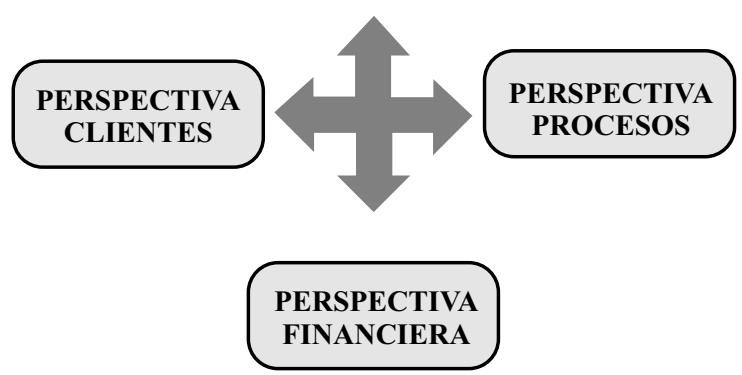

\section{MODELO DE GESTIÓN}

En el año 2001, luego de asumir una nueva jefatura este servicio tras 12 años de un mismo jefe, se inició un proceso de planificación estratégica participativo que significó el diseño del Plan Estratégico y en consecuencia la conformación y uso del BSC. El Servicio de Pediatría del Hospital Regional Rancagua tiene una dotación de 60 funcionarios, y 44 camas, de las cuales 4 son camas críticas; egresa un promedio de 2000 pacientes por año, y el perfil epidemiológico corresponde a un servicio de pediatría de pacientes agudos con la estacionalidad similar 
Figura 2. Mapa estratégico

al resto del país, es decir, predominio de enfermedades respiratorias en los meses de invierno.

A contar del año 2003, luego de definidas la Visión, Misión y Valores del servicio, se consolida este proceso, aplicándose estrategias de gestión modernas que llevaron a establecer un estilo directivo diferente al resto del hospital cuyos resultados se presentan a continuación.

\section{Declaraciones estratégicas}

Fueron definidas luego de un proceso que contó con la participación de todos los integrantes del equipo humano que significó la capacitación en Planificación Estratégica dirigida por la jefatura y el desarrollo del proceso estratégico posterior.

a. Visión: creada por el líder del equipo, se explicitó con la siguiente declaración: Contribuir a la felicidad y el desarrollo de los niños de la Sexta Región.

b. Misión: construida en una jornada de planificación con la más amplia participación de los miembros del equipo, explicitada así : Recuperar la salud de los niños ingresados al servicio en el menor tiempo, mediante el trabajo en equipo, aplicando las mejores técnicas diagnósticas y terapéuticas en un ambiente cordial y con la participación activa de los padres.

c. Valores: declarados en forma unánime, son: Lealtad - Honestidad - Solidaridad Colaboración - Sensibilidad

\section{Mapa estratégico}

Es el conjunto de los objetivos estratégicos interrelacionados y direccionados por la Visión o norte estratégico, en las 4 perspectivas descritas en el modelo y que son: perspectiva de las personas, perspectiva del proceso interno, perspectiva del cliente y perspectiva financiera.

a. Perspectiva de las personas: Se consideró

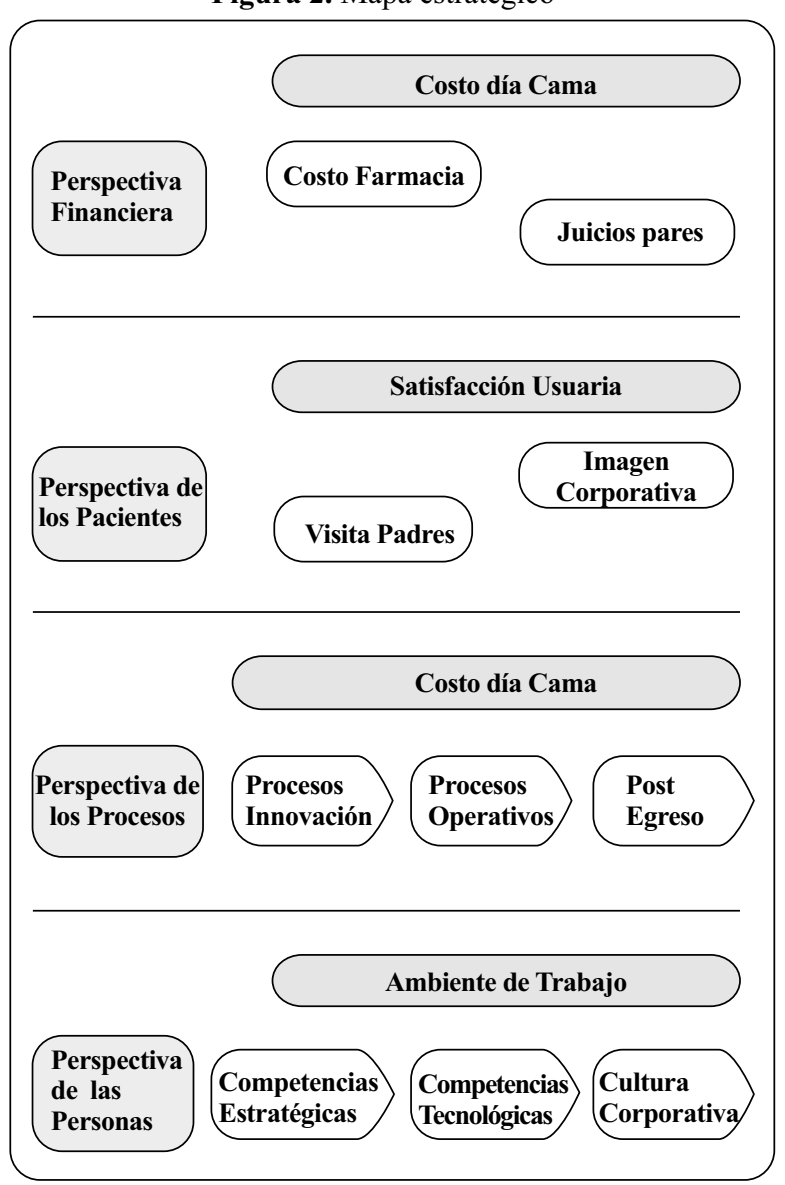

como la perspectiva clave en el proceso estratégico del Servicio de Pediatría y significó un trabajo intensivo del líder con los médicos, enfermeras, técnicos paramédicos, auxiliares y secretaria, definiendo objetivos que exigieran desarrollar competencias estratégicas y tecnológicas, estableciendo una cultura corporativa y así un ambiente de trabajo apto para la aplicación de estrategias adecuadas.

i. En las competencias estratégicas, se definió la necesidad de cambiar el estilo de jefatura de las enfermeras, capacitándolas en motivación, liderazgo, comunicación.

ii. En el estamento técnico paramédico, el objetivo fue modificar la actitud de pasividad frente al proceso diagnóstico y terapéutico y generar una actitud proactiva, que incluyó la 
participación en el control de la gestión y en el diseño de nuevos procedimientos.

iii. Los auxiliares fueron incorporados en el objetivo de desarrollar nuevas competencias en áreas como la mantención y reparación de infraestructura y también tomaron conocimiento de la gestión del servicio.

iv. La secretaria debería desarrollar nuevas competencias en computación y gestión que hicieron posible mejorar su expedición en el manejo de la información y en la comunicación formal del servicio.

v. Los médicos deberían adquirir competencias en el área del análisis epidemiológico, desarrollo de guías clínicas y definición de los códigos de las enfermedades, alcanzando mayor precisión diagnóstica.

vi. Competencias tecnológicas: Médicos, enfermeras y técnicos paramédicos asumieron la necesidad de incorporar la informática como herramienta de gestión y análisis.

b. Perspectiva del proceso interno: se trató de identificar los procesos en que el Servicio de Pediatría debía buscar la excelencia operativa. Es decir, dentro de la cadena de valor se definieron 3 tipos de procesos a desarrollar.

i. Procesos de innovación: que consideraron el análisis del uso de camas de otros servicios de pediatría de hospitales públicos, realizando benchmarking con hospitales de características similares al Hospital de Rancagua. Además, se inició la actividad de auditoría de los egresos mediante un experto que definió los estándares.

ii. Procesos operativos: la producción del servicio, consistente en egresos y días cama se debería optimizar, buscando acortar las estadías intrahospitalarias y entregar excelencia con un enfoque de calidad que consideró la disminución de las infecciones y accidentes nosocomiales. Además, los médicos deberían diseñar las guías clínicas de las patologías principales, lo que significó estandarización de los procesos de diagnóstico y tratamiento y la consecuente optimización del uso de los recursos.

iii. Procesos post-hospitalización: Se hizo necesario implementar un policlínico de control de altas que permitió dar altas en menor tiempo con la seguridad del control oportuno y limitar los eventuales reingresos.

c. Perspectiva de los pacientes y comunidad: Se buscó mejorar la posición del Servicio de Pediatría en el mercado de los prestadores de atención de salud de la región, identificando las ventajas competitivas del servicio y asegurando su reconocimiento en la comunidad regional.

i. Imagen: Diseñar una imagen corporativa que identifique al servicio en todas las actividades intra y extrahospitalarias, especialmente del ámbito comunidad profesional y en los medios de comunicación regionales.

ii. Visitas de padres: Enfrentar la separación de los pacientes-niños de sus padres con la implementación de una visita diaria de 7 horas diarias, con un diseño de acogida que aprovecha de explicitar los deberes de la familia de los niños hospitalizados.

iii. Satisfacción usuaria: Medirla mediante la aplicación de una encuesta de satisfacción usuaria, la que debería ser diseñada, que se tabula regularmente y que permite evaluar los aspectos más sensibles de calidad de la atención.

iv. Investigación clínica y epidemiológica: Considerar la participación en todos los congresos chilenos de Pediatría y en todas aquellas actividades de las sociedades científicas que marquen posición en la producción del servicio.

d. Perspectiva financiera: Debido a que el servicio no maneja presupuesto propio, se consideró monitorear el costo de farmacia que representa, luego del gasto en personal, el ítem más grande de gasto. 
i. Costo mensual de fármacos: definido el gasto total en este ítem. ii. Se revisó la pertinencia del uso de los fármacos que representa el $80 \%$ del gasto, mediante un análisis de pares.

Tabla 1. Indicadores de la perspectiva de las personas

\section{COMPETENCIAS ESTRATÉGICAS}

\begin{tabular}{lccc}
\hline Objetivo & Indicador & Inicial 2000 & Actual 2007 \\
\hline $\begin{array}{l}\text { Capacitación de enfermeras } \\
\text { en Liderazgo, Comunicación } \\
\text { y Motivación }\end{array}$ & $\begin{array}{c}\text { \% enfermeras } \\
\text { capacitadas }\end{array}$ & $10 \%$ & $90 \%$ \\
\hline $\begin{array}{l}\text { Participación en el } \\
\text { control de gestión }\end{array}$ & $\begin{array}{c}\mathrm{N}^{\mathrm{o}} \text { reuniones } \\
\text { de evaluación con los } \\
\text { delegados de turno }\end{array}$ & 0 & 10 en el año \\
$\begin{array}{l}\text { Actividades } \\
\text { de mantención }\end{array}$ & $\begin{array}{c}\mathrm{N}^{\circ} \text { tareas } \\
\text { específicas realizadas }\end{array}$ & 1 trimestral & 1 mensual \\
\hline
\end{tabular}

COMPETENCIAS TECNOLÓGICAS

\begin{tabular}{lccc}
\hline Objetivo & Indicador & Inicial 2000 & Actual 2007 \\
\hline $\begin{array}{l}\text { Mejorar calidad } \\
\text { diagnóstica de } \\
\text { médicos }\end{array}$ & $\begin{array}{l}\text { \% de diagnóstico } \\
\text { de egresos del Cap. } \\
\text { XVIII del CIE-10 }\end{array}$ & $3,5 \%$ & $2,1 \%$ \\
\cline { 2 - 4 } & $\begin{array}{c}\mathrm{N}^{\mathrm{o}} \text { patologías } \\
\text { con guía clínica }\end{array}$ & 0 & 41 \\
\hline $\begin{array}{l}\text { Incorporación de } \\
\text { herramientas de } \\
\text { informática }\end{array}$ & $\begin{array}{c}\text { \% de formularios } \\
\text { con plantilla }\end{array}$ & 0 & $80 \%$ \\
\hline $\begin{array}{l}\text { Incrementar análisis } \\
\text { bioestadístico }\end{array}$ & $\begin{array}{l}\text { \% médicos } \\
\text { capacitados }\end{array}$ & $5 \%$ & $80 \%$ \\
\hline
\end{tabular}

\section{CULTURA CORPORATIVA}

\section{Objetivo}

Monitores de resultados

Mejorar calidad de la información

Aumentar la participación en la gestión

Indicador

Inicial 2000

Actual 2007

$\mathrm{N}^{\mathrm{o}}$ de reuniones de evaluación por estamento

$$
\% \text { de diagnósticos }
$$

errados en Boletín de Egreso

$$
\mathrm{N}^{\mathrm{o}} \text { planes de acción }
$$

$30 \%$
$1 \%$

4 en el año generados participativamente

10




\section{Cuadro de mando integral}

Cumple la función de dar estructura a los componentes estratégicos, los integra en un formato que permite tener toda la información estratégica del Servicio. Para esto, se debió construir los indicadores asociados a los objetivos en cada perspectiva previamente mostrada.

Tabla 2. Indicadores de la perspectiva de los procesos internos

\begin{tabular}{|c|c|c|c|}
\hline \multicolumn{4}{|c|}{ PROCESOS DE INNOVACIÓN } \\
\hline Objetivo & Indicador & Inicial 2000 & Actual 2007 \\
\hline Benchmarking & $\mathrm{N}^{\mathrm{o}}$ de benchmarks & 0 & 40 \\
\hline Auditoría de egresos & $\%$ de egresos auditados & 0 & $100 \%$ \\
\hline \multicolumn{4}{|c|}{ PROCESOS OPERATIVOS } \\
\hline Objetivo & Indicador & Inicial 2000 & Actual 2007 \\
\hline Acortar las estadías & Promedio días estada & 6,3 días & 3,9 días \\
\hline $\begin{array}{l}\text { Disminuir Infecciones } \\
\text { Intrahospitalarias }\end{array}$ & $\begin{array}{l}\text { Tasa Neumonía asociada } \\
\text { a ventilación mecánica }\end{array}$ & $31,3 \%$ & $13,5 \%$ \\
\hline $\begin{array}{l}\text { Implementar } \\
\text { controles de alta }\end{array}$ & $\mathrm{N}^{\mathrm{o}}$ controles & 0 & 433 (año 2006) \\
\hline \multicolumn{4}{|c|}{ PROCESOS POST- EGRESO } \\
\hline Objetivo & Indicador & Inicial 2000 & Actual 2007 \\
\hline Disminuir los reingresos & $\%$ de reingresos & ¿? & $6,6 \%$ \\
\hline
\end{tabular}

Se muestran algunos indicadores que forman el BSC de nuestro servicio con la evolución en el período $2001-2007$.

Se aprecia los indicadores usados en la perspectiva de las personas, destacando el esfuerzo realizado en capacitación en todos los estamentos, la confección de guías clínicas y especialmente la participación en la gestión del servicio.

En la perspectiva de los procesos internos, se hizo énfasis en la gestión de las camas, acortando las estadías, asociado a la implementación de controles de alta. Se usaron los benchmarks para definir las metas a lograr. Además, se trabajó en planes de manejo de las infecciones intrahospitalarias, y se presenta el resultado en las neumonías asociadas a ventilación mecánica. Al no existir registro de los reingresos, se comienza con este indicador de manera de poder establecer la "normalidad" para nuestro servicio.

Los indicadores de la perspectiva de los pacientes y la comunidad incluyeron la creación de la imagen corporativa y especialmente medir la satisfacción usuaria mediante la aplicación de una encuesta que se validó inicialmente.

La perspectiva financiera, la menos relevante dado que no se maneja presupuesto propio aún, consideró iniciar el conocimiento del gasto de farmacia y más importante, conocer la pertinencia de este gasto. 
Tabla 3. Indicadores de la perspectiva de los pacientes y comunidad

\begin{tabular}{|c|c|c|c|}
\hline \multicolumn{4}{|c|}{ IMAGEN CORPORATIVA } \\
\hline Objetivo & Indicador & Inicial 2000 & Actual 2007 \\
\hline Crear imagen corporativa & Imagen corporativa & 0 & Existe \\
\hline \multicolumn{4}{|c|}{ VISITA DE PADRES } \\
\hline Objetivo & Indicador & Inicial 2000 & Actual 2007 \\
\hline Implementar visita de padres & $\mathrm{N}^{\mathrm{o}}$ horas de visita & 0,8 horas/día & 7 horas/día \\
\hline \multicolumn{4}{|c|}{ SATISFACCIÓN USUARIA } \\
\hline Objetivo & Indicador & Inicial 2000 & Actual 2007 \\
\hline Diseñar y aplicar encuesta & 0 de egresos con encue & sta & $80 \%$ \\
\hline Mejorar satisfacción usuaria & Nota promedio final & ¿? & 6,6 \\
\hline \multicolumn{4}{|c|}{ INVESTIGACIÓN Y POSICIONAMIENTO } \\
\hline Objetivo & Indicador & Inicial 2000 & Actual 2007 \\
\hline $\begin{array}{l}\text { Realizar investigación } \\
\text { y participación en } \\
\text { congresos científicos }\end{array}$ & $\begin{array}{c}\mathrm{N}^{\circ} \text { trabajos } \\
\text { expuestos por año }\end{array}$ & 0 & 9 (año 2006) \\
\hline
\end{tabular}

Tabla 4. Indicadores de la perspectiva financiera

\begin{tabular}{|lcccc|}
\hline \multicolumn{4}{|c|}{ GASTOS FARMACIA } \\
\hline Objetivo & Indicador & Inicial 2000 & Actual 2007 \\
\hline Conocer gasto de farmacia & Gasto mensual & $i ?$ & Informe mensual \\
\hline $\begin{array}{l}\text { Pertinencia uso } \\
\text { fármacos grupo A }\end{array}$ & $\begin{array}{c}\text { \% de pertinencia } \\
\text { según juicio }\end{array}$ & $i ?$ & 90 \\
\hline
\end{tabular}

\section{DISCUSIÓN}

Exponemos la aplicación de un modelo de gestión, Cuadro de Mando Integral, en una organización particular, en un hospital público y regional. Este modelo ha permitido mejorar nuestro desempeño y es una demostración de que es posible de aplicar en una empresa de servicios de atención de salud ${ }^{6,7}$.

El Servicio de Pediatría del Hospital Regional Rancagua ha vivido un interesante y desafiante período entre los años 2001 a 2007, que ha significado cambiar la forma de "hacer las cosas" para todos quienes trabajamos en él.

Los procesos de cambio realizados han estado centrados en las personas que formamos el equipo y en la búsqueda de excelencia en la atención de nuestros pequeños pacientes y sus familias. El cambio de jefatura del año 2001 fue difícil y demandó demostrar con hechos que el Servicio podía mejorar. Pero, más importante, fue la evidente preocupación por los funcionarios 
como personas, con sus fortalezas y debilidades y desde el detalle del saludo personalizado a todos, al reconocimiento del resto del hospital como un servicio "diferente" son las características destacadas que son apreciadas por todos.

Un mensaje trascendente y verdaderamente internalizado es que en nuestro servicio "todos valen" y la responsabilidad con los resultados es por lo tanto de todos.

Hemos puesto especial empeño en mostrar la importancia de las iniciativas de todos los estamentos, crear un ambiente amable y mostrar compromiso con las declaraciones estratégicas que juntos declaramos.

La gestión de un servicio clínico en un ambiente de carencia general como es nuestro hospital, supone esfuerzo pero por sobre todo trabajo en equipo y compromiso. Ambos conceptos son válidos en nuestro Servicio.

\section{REFERENCIAS}

1. KAPLAN RS, NORTON DP. The Balanced Scorecard. Boston: Harvard Business School Press; 1996.

2. Curso Metodología del Balance Scorecard. Les Halles 2007.
3. ASENJO M.A. Gestión diaria del hospital. Ed. Masson 2001.

4. PELL A. Administre a su personal. Ed. Prentice Hall. 1996.

5. BODENHEIMER T, BOJESTIG M, HENRIKS G. Making Systemwide Improvements in Health Care: Lessons From Jönköping County, Sweden. Q Manage Health Care. Vol. 16, Nº1, 10:15

6. INAMDAR N, KAPLAN R. Applying the Balanced Scorecard in Healthcare Provider Organizations. Journal of Healthcare Management 47:3 May/June 2002.

7. VILLALBI J, GUIX J, CASAS C, BORRELL C, DURAN J, ARTAZCOZ L, CAMPRUBÍ E, CUSÍ M, RODRÍGUEZ-MONTUQUÍN P, ARMENGOL J, JIMÉNEZ G. El Cuadro de Mando Integral como instrumento de dirección en una organización de salud pública. Gac Sanit. 2007;21 (1): 60-5.

Recepción: 11 septiembre 2007 Aprobación: 15 noviembre 2007

Usted puede comentar éste y otros artículos publicados en la Revista Chilena de Salud Pública, enviando un correo electrónico a revistasp@med.uchile.cl 